Supporting Information for

\title{
Smart Superstructures with Ultrahigh pH-Sensitivity for Targeting Acidic Tumor Microenvironment: Instantaneous Size Switching and Improved Tumor Penetration
}

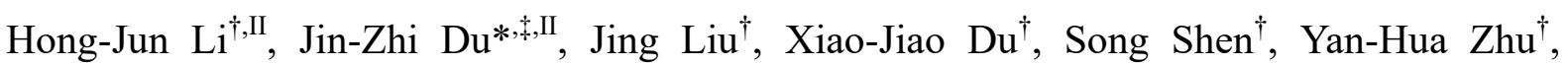
Xiaoyan Wang ${ }^{\S}$, Xiaodong $\mathrm{Ye}^{\S}$, Shuming Nie, ${ }^{*, \downarrow}$, and Jun Wang ${ }^{*, \dagger, \$, \perp}$

${ }^{\dagger}$ CAS Center for Excellence in Nanoscience, School of Life Sciences and Medical Center, University of Science and Technology of China, Hefei, Anhui 230027, China

Department of Biomedical Engineering, Emory University and Georgia Institute of Technology, Atlanta, Georgia 30322, United States

${ }^{\S}$ Hefei National Laboratory for Physical Sciences at the Microscale, Hefei, Anhui 230027, China

${ }^{\perp}$ Innovation Center for Cell Signaling Network, University of Science and Technology of China, Hefei, Anhui 230027, China 
Materials and Reagents. The fourth generation poly(amidoamine) (PAMAM) dendrimer was purchased from Weihai CY Dendrimer Technology Co., Ltd. (Shandong, China). Cisplatin was purchased from Shandong Boyuan Pharmaceutical Co., Ltd. (Shandong, China) and the cisplatin prodrug $c, c, t-\left[\mathrm{Pt}\left(\mathrm{NH}_{3}\right)_{2} \mathrm{Cl}_{2}(\mathrm{OH})\left(\mathrm{O}_{2} \mathrm{CCH}_{2} \mathrm{CH}_{2} \mathrm{CO}_{2} \mathrm{H}\right)\right]$ was prepared as previously described ${ }^{1}$. Platinum $(\mathrm{Pt})$ conjugated PAMAM (PAMAM/Pt) was synthesized by an amidation reaction between PAMAM and $c, c, t-\left[\mathrm{Pt}\left(\mathrm{NH}_{3}\right)_{2} \mathrm{Cl}_{2}(\mathrm{OH})\left(\mathrm{O}_{2} \mathrm{CCH}_{2} \mathrm{CH}_{2} \mathrm{CO}_{2} \mathrm{H}\right)\right]^{2}$ 1-(3-Dimethylaminopropyl)-3-ethylcarbodiimide hydrochloride $\quad$ (EDC.HCl), $\mathrm{N}$-hydroxysuccinimide (NHS) were purchased from Aladdin Industrial, Inc. (Shanghai, China). The macroinitiator $\mathrm{mPEG}_{113}-\mathrm{CPDB}$ was synthesized as previously described ${ }^{3}$. The monomer 2-azepane ethyl methacrylate (AEMA) was synthesized as previously described ${ }^{4}$. $N$-(ع-Maleimidocaproyloxy)succinimide ester and azobisisobutyronitrile (AIBN) were purchased from Sigma-Aldrich Co., LLC. (Shanghai, China). 2-Cyclohexylethanol and methacryloyl chloride were purchased from Alfa Aesar Chemical Co., Ltd. (China). Other chemicals were analytical grade and used as received.

RPMI-1640 medium was purchased from GIBCO (Grand Island, USA), and fetal bovine serum (FBS) was purchased from ExCell Bio (Shanghai, China). Trypsin-EDTA (0.25\%) and Penicillin-streptomycin solutions were purchased from MesGen Biotech. (Shanghai, China). The antibody to PECAM-1 and FITC-labeled secondary antibody were purchased from Santa Cruz Biotechnology, Inc. (USA). The sulfo-Cy5 NHS probe was purchased from Lumiprobe Corporation (Hallandale, USA). Agarose was obtained from Sigma-Aldrich Co., LLC. (Shanghai, China). The dialysis bags with series of cut-off molecular weight were purchased from Shanghai Green Bird Science \& Technology Development Co., LTD (Shanghai, China). 
Characterizations. ${ }^{1} \mathrm{H}$ NMR spectra were recorded on a Bruker AV400 NMR spectrometer (Bruker Corporation, Switzerland) using $\mathrm{CDCl}_{3}$ as the solvent. The gel permeation chromatography (GPC) system is composed of a Waters 1515 pump, and a Waters 2414 refractive index detector, equipped with Waters styragel high-resolution columns (HR4, HR2, HR1, and HR0.5; effective molecular-weight range: 5000-500,000; 500-20,000; 100-5,000; and 0-1,000; respectively) at $30^{\circ} \mathrm{C}$. Chloroform (HPLC grade, DUKSAN, stabilized with amylene) was used as the mobile phase and delivered at a flow rate of $1.0 \mathrm{~mL} \mathrm{~min}{ }^{-1}$. Gel filtration chromatography was performed on a Waters high performance liquid chromatography (HPLC) system, equipped with a Waters 1525 binary pump, Waters 2424 evaporative light-scattering detector, using Waters Ultrahydrogel ${ }^{\mathrm{TM}} 1000$ as the column at $30{ }^{\circ} \mathrm{C}$. Pure water was used as the mobile phase with a flow rate of $1.0 \mathrm{~mL} / \mathrm{min}$. HPLC system was equipped with a Waters 1525 binary pump, Waters 2424 evaporative light-scattering detector and $\mathrm{C}_{18}$ reverse phase column. HPLC grade acetonitrile/water (50/50, $\mathrm{v} / \mathrm{v}$ ) was used as the mobile phase at $30^{\circ} \mathrm{C}$ with a flow rate of $1.0 \mathrm{~mL} / \mathrm{min}$. Breeze software was used for data analysis. Platinum content was measured on an X Series 2 Inductively Coupled Plasma Mass Spectrometer (ICP-MS, Thermo Fisher Scientific, USA). Particle size and zeta potential measurements were conducted using a zeta potential analyser with dynamic light-scattering capability on a Malvern Zetasizer Nano ZS90 (Malvern Instruments Ltd., UK) with He-Ne laser $(633 \mathrm{~nm})$ and $90^{\circ}$ collecting optics. Data were analysed using Malvern Dispersion Technology Software 7.02. Morphology of nanoparticles was examined by JEOL-2010 transmission electron microscopy (TEM, JEOL Co. Ltd., Tokyo, Japan) at an accelerating voltage of $200 \mathrm{kV}$. The sample was prepared by pipetting a drop of the aqueous 
solution of nanoparticles $\left(0.5 \mathrm{mg} \mathrm{mL}^{-1}\right)$ onto a 230 mesh copper grid coated with carbon. The time scale of $\mathrm{pH}$-dependent size change was measured by stopped-flow instrument.

\section{Preparation of PEG-b-PAEMA-PAMAM/Pt (Scheme S1)}

(1) Preparation of PEG- $b$-PAEMA. PEG- $b$-PAEMA was synthesized by RAFT polymerization. Briefly, $\mathrm{mPEG}_{113}$-CPDB (0.20 g, 1.0 eqv), AEMA (1.07 g, 130 eqv), and AIBN (3.1 mg, 0.33 eqv) were dissolved in anhydrous dioxane. After freezing and thawing several times to remove oxygen, the mixture was stirred at $75{ }^{\circ} \mathrm{C}$ for $10 \mathrm{~h}$, and then precipitated into cold hexane. The precipitate was collected by filtration. After drying overnight in vacuum, the product was obtained as a yellow solid in 50\% yield. ${ }^{1} \mathrm{H}$ NMR (400 $\left.\mathrm{MHz}, \mathrm{CDCl}_{3}, \mathrm{ppm}\right): \delta 4.02(\mathrm{~m}, 180 \mathrm{H}), 3.64(\mathrm{~m}, 454 \mathrm{H}), 3.38(\mathrm{~s}, 3 \mathrm{H}), 2.83-2.63(\mathrm{~m}, 540 \mathrm{H})$, 2.02-1.76 (m, 180H), $1.61(\mathrm{~m}, 720 \mathrm{H}), 1.10-0.82(\mathrm{~m}, 270 \mathrm{H})$. The degree of polymerization of PAEMA block was 90 as determined according to ${ }^{1} \mathrm{H}$ NMR spectrum. Its molecular weight distribution was 1.21 as determined by GPC.

(2) Preparation of PEG-b-PAEMA-PAMAM/Pt. The terminal thiobenzoylthio group was transferred into thiol to obtain sulfhydryl-terminated PEG- $b$-PAEMA according to previously reported literature ${ }^{5}$. The sulfhydryl-terminated PEG- $b$-PAEMA $(0.300 \mathrm{~g}, 1.0$ eqv) was dissolved in anhydrous dioxane under nitrogen atmosphere, and then $N$-( $\varepsilon$-maleimidocaproyloxy)succinimide ester $(7.7 \mathrm{mg}, 2.0 \mathrm{eqv})$ was added into the solution. The mixture was stirred overnight at room temperature. The product PEG- $b$-PAEMA-NHS was purified by precipitating the mixture into ether and drying under vacuum, and obtained as a white solid in 90\% yield. Then, PAMAM/Pt (50 mg, 1.0 eqv) and PEG- $b$-PAEMA-NHS (266 mg, 3.0 eqv) were dissolved in anhydrous dimethylformamide (DMF) and reacted at 30 
${ }^{\circ} \mathrm{C}$ for $24 \mathrm{~h}$. The product was further purified by dialysis $(\mathrm{MWCO}=50,000 \mathrm{Da})$ in DMF. The solvent was removed under vacuum and the product was lyophilized and obtained as a white solid (yield: $80 \%$ ). The Pt content in PAMAM/Pt was 2.4\% determined by ICP-MS and 2.1 PEG- $b$-PAEMA polymer chains were attached to PAMAM/Pt on average.

\section{Preparation of PEG-b-PCHMA-PAMAM/Pt (Scheme S2)}

(1) Preparation of 2-cyclohexylethyl methacrylate (CHMA). 2-Cyclohexylethanol (5.00 g, 1.0 eqv) and triethylamine (3.95 g, 1.0 eqv) were dissolved in anhydrous tetrahydrofuran and stirred for $10 \mathrm{~min}$ at $0{ }^{\circ} \mathrm{C}$. Then methacryloyl chloride (4.06 g, $\left.1.0 \mathrm{eqv}\right)$ was added and the mixture was stirred for $10 \mathrm{~h}$ at $0{ }^{\circ} \mathrm{C}$. After removing the salt, the liquid was collected and concentrated in the vacuum. The product was obtained as a colorless liquid through distillation under vaccum. Yield: 80\%. ${ }^{1} \mathrm{H}$ NMR (400 MHz, $\left.\mathrm{CDCl}_{3}, \mathrm{ppm}\right): \delta 6.12(\mathrm{~s}, 1 \mathrm{H})$, $5.58(\mathrm{~s}, 1 \mathrm{H}), 4.25(\mathrm{t}, 2 \mathrm{H}), 1.95(\mathrm{~s}, 3 \mathrm{H}), 1.78-1.53(\mathrm{~m}, 8 \mathrm{H}), 1.19(\mathrm{~m}, 3 \mathrm{H})$ and $0.95(\mathrm{~m}, 2 \mathrm{H})$. (2) Preparation of PEG-b-PCHMA. PEG- $b$-PCHMA was synthesized by RAFT polymerization with the same method as PEG- $b$-PAEMA, by replacing AEMA with CHMA. The product was obtained as a yellow solid in 50\% yield. ${ }^{1} \mathrm{H}$ NMR (400 $\left.\mathrm{MHz}, \mathrm{CDCl} 3, \mathrm{ppm}\right)$ : $\delta 4.02(\mathrm{~m}, 180 \mathrm{H}), 3.64(\mathrm{~m}, 454 \mathrm{H}), 3.38(\mathrm{~s}, 3 \mathrm{H}), 2.00-1.60(\mathrm{~m}, 689 \mathrm{H}), 1.60-1.45(\mathrm{~m}, 204 \mathrm{H})$, 1.43-1.11 (m, $460 \mathrm{H}), 1.08-0.80(\mathrm{~m}, 457 \mathrm{H})$. The degree of polymerization of PCHMA block was 90 as determined according to the ${ }^{1} \mathrm{H}$ NMR spectrum. Its molecular weight distribution was 1.19 as determined by GPC.

(3) Preparation of PEG-b-PCHMA-PAMAM/Pt. The $\mathrm{pH}$-insensitive PEG- $b$-PCHMA-PAMAM/Pt was obtained via the same method described as the preparation of PEG- $b$-PAEMA-PAMAM/Pt. The Pt content in PAMAM/Pt was $2.4 \%$ determined by 
ICP-MS and 2.2 PEG- $b$-PCHMA polymer chains were attached to PAMAM/Pt on average.

\section{Reference}

(1) Dhar, S.; Daniel, W. L.; Giljohann, D. A.; Mirkin, C. A.; Lippard, S. J. Polyvalent Oligonucleotide Gold Nanoparticle Conjugates as Delivery Vehicles for Platinum(Iv) Warheads. J. Am. Chem. Soc. 2009, 131, 14652-14653.

(2) Li, H. J.; Du, J. Z.; Du, X. J.; Xu, C. F.; Sun, C. Y.; Wang, H. X.; Cao, Z. T.; Yang, X. Z.; Zhu, Y. H.; Nie, S. M.; Wang, J. Stimuli-Responsive Clustered Nanoparticles for Improved Tumor Penetration and Therapeutic Efficacy. Proc. Natl. Acad. Sci. U. S. A. 2016, 113, 4164-4169.

(3) Zhang, P. P.; Jiang, K.; Ye, C. N.; Zhao, Y. L. Facile Synthesis of V-Shaped Copolymer Brushes Grafted onto the Surface of Graphene Oxide via Coupling Reactions. Chem. Commun. 2011, 47, 9504-9506.

(4) Ma, X. P.; Wang, Y. G.; Zhao, T.; Li, Y.; Su, L. C.; Wang, Z. H.; Huang, G.; Sumer, B. D.; Gao, J. M. Ultra-pH-Sensitive Nanoprobe Library with Broad pH Tunability and Fluorescence Emissions. J. Am. Chem. Soc. 2014, 136, 11085-11092.

(5) Li, M.; De, P.; Gondi, S. R.; Sumerlin, B. S. End Group Transformations of Raft-Generated Polymers with Bismaleimides: Functional Telechelics and Modular Block Copolymers. J. Polym. Sci., Part A-1: Polym. Chem. 2008, 46, 5093-5100. 

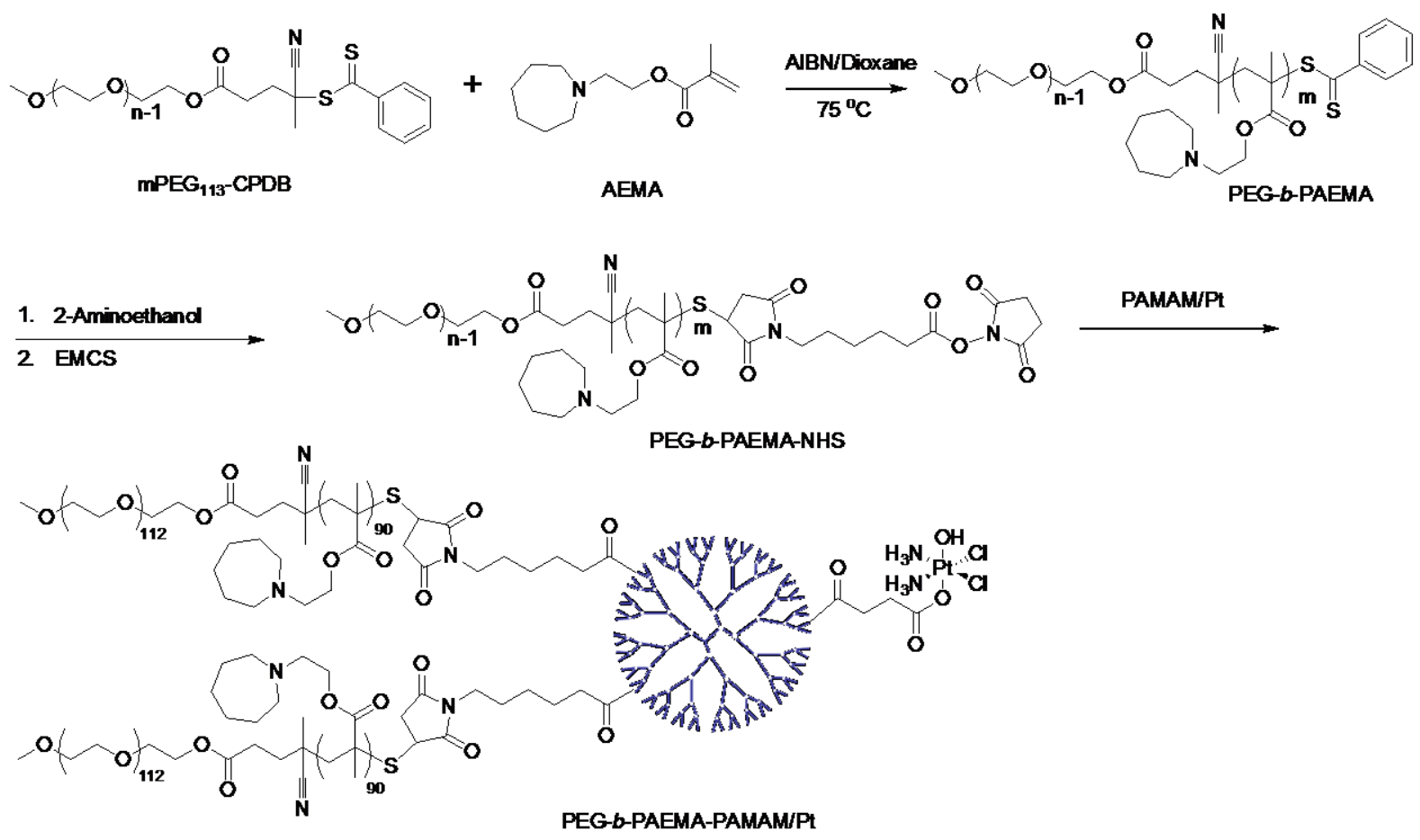

Scheme S1. Synthetic procedures of PEG- $b$-PAEMA-PAMAM/Pt. EMCS: $N$-(ع-maleimidocaproyloxy)succinimide ester; AIBN: azobisisobutyronitrile. 

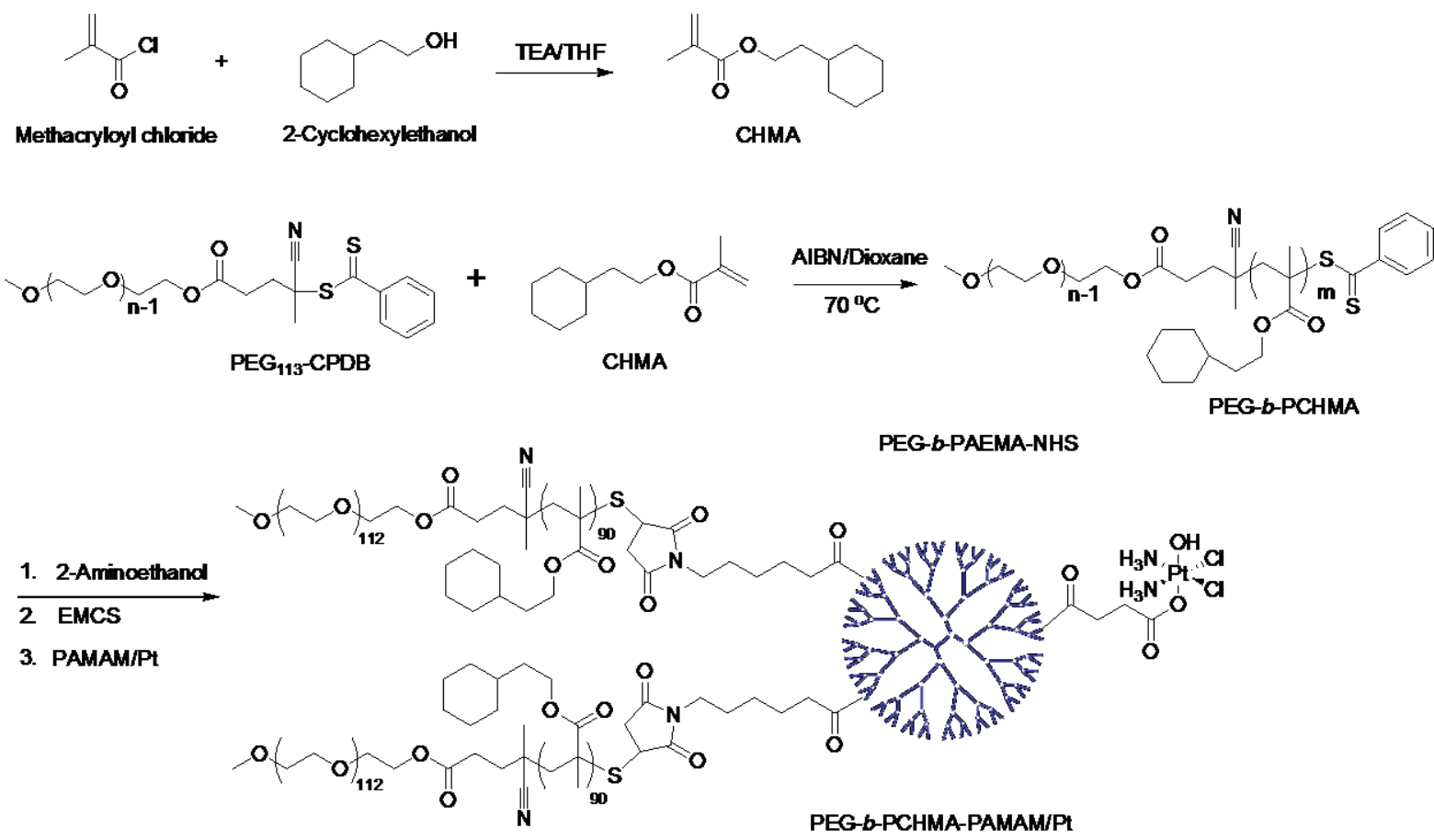

Scheme S2. Synthetic procedures of PEG- $b$-PCHMA-PAMAM/Pt. EMCS: $N$-(E-maleimidocaproyloxy)succinimide ester; AIBN: azobisisobutyronitrile; TEA: Triethylamine; THF: Tetrahydrofuran. 


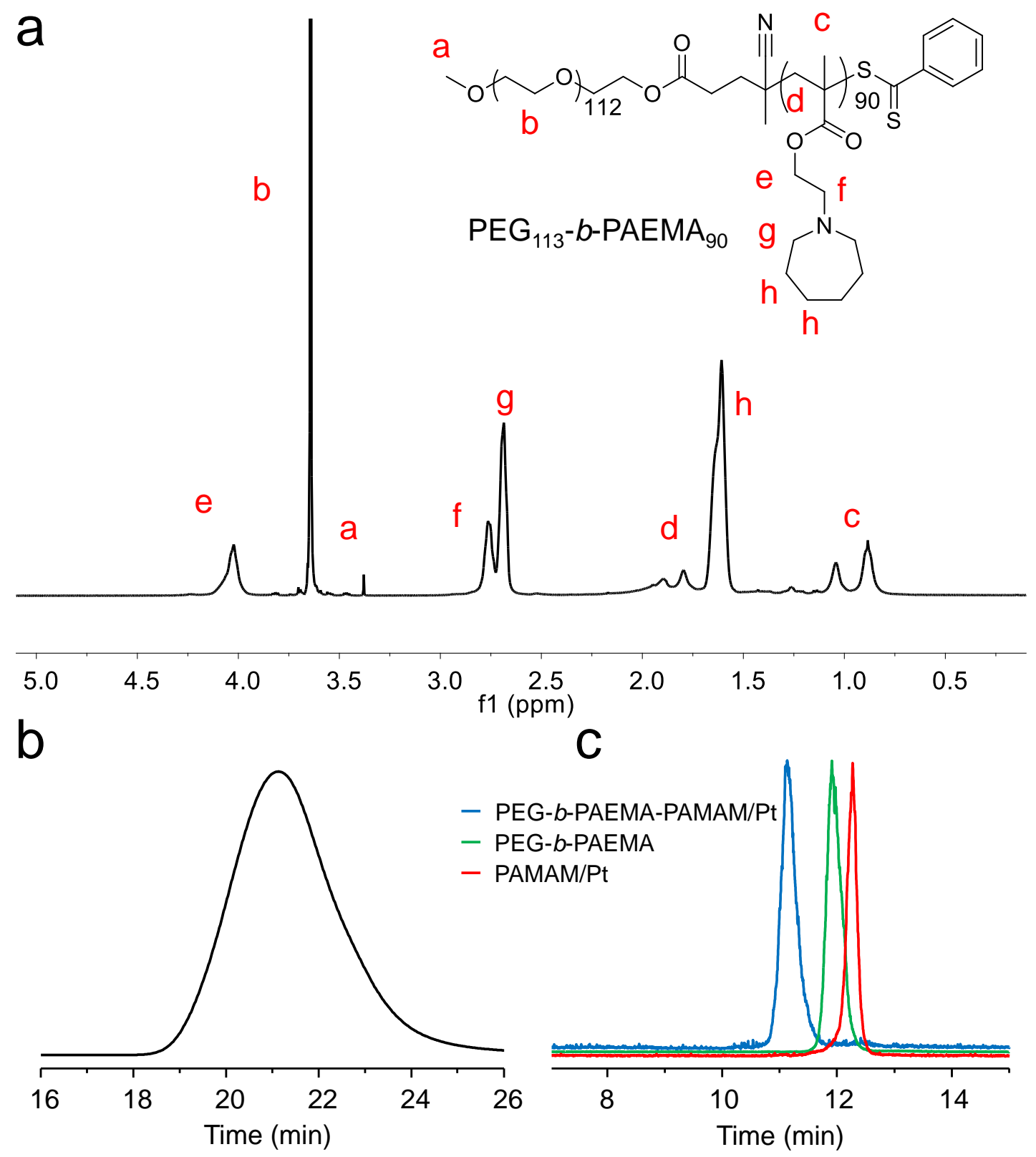

Figure S1. (a) ${ }^{1}$ H NMR spectrum and (b) GPC profile of of PEG- $b$-PAEMA. (c) Gel filtration chromatography profiles of PAMAM/Pt, PEG- $b$-PAEMA and PEG- $b$-PAEMA-PAMAM/Pt using water as the eluent. PEG- $b$-PAEMA and PEG- $b$-PAEMA-PAMAM/Pt were protonated with $\mathrm{HCl}$ before measurement. 

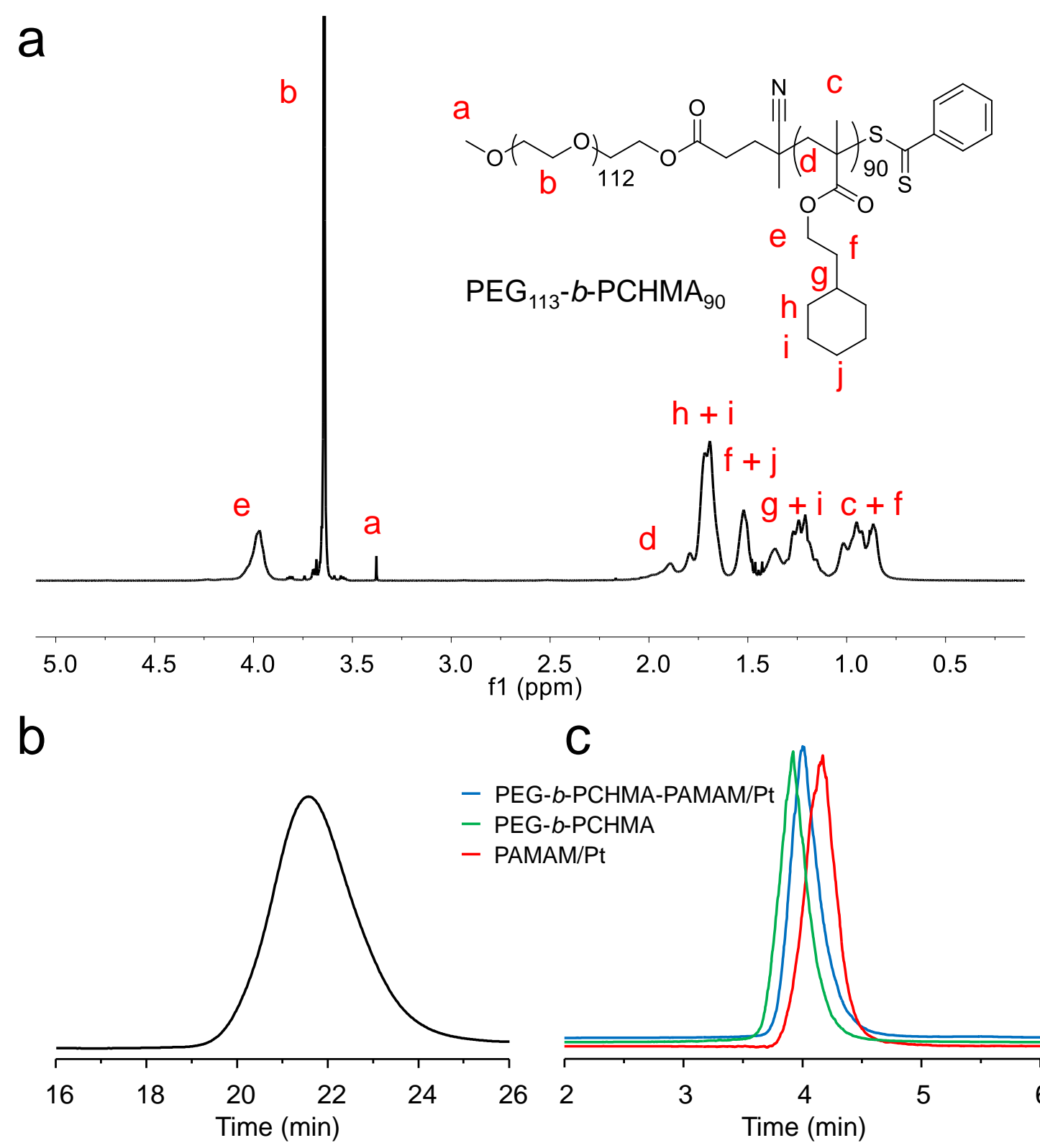

C

Figure S2. (a) ${ }^{1} \mathrm{H}$ NMR spectrum and (b) GPC profile of of PEG- $b$-PCHMA. (c) HPLC profiles of PAMAM/Pt, PEG- $b$-PCHMA and PEG- $b$-PCHMA-PAMAM/Pt. 


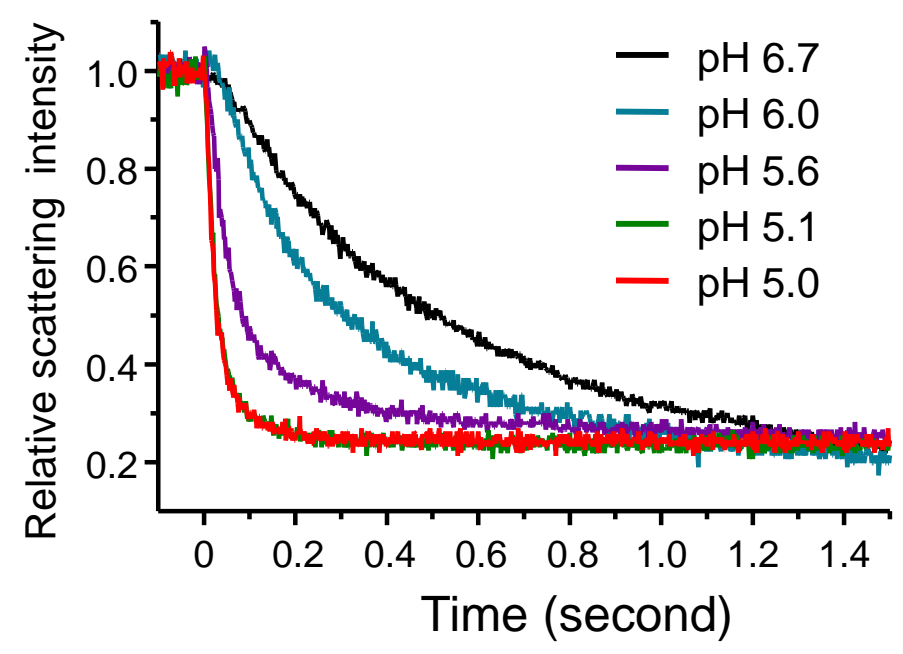

Figure S3. pH-Dependent response time of SCNs/Pt measured by stopped-flow instrument.
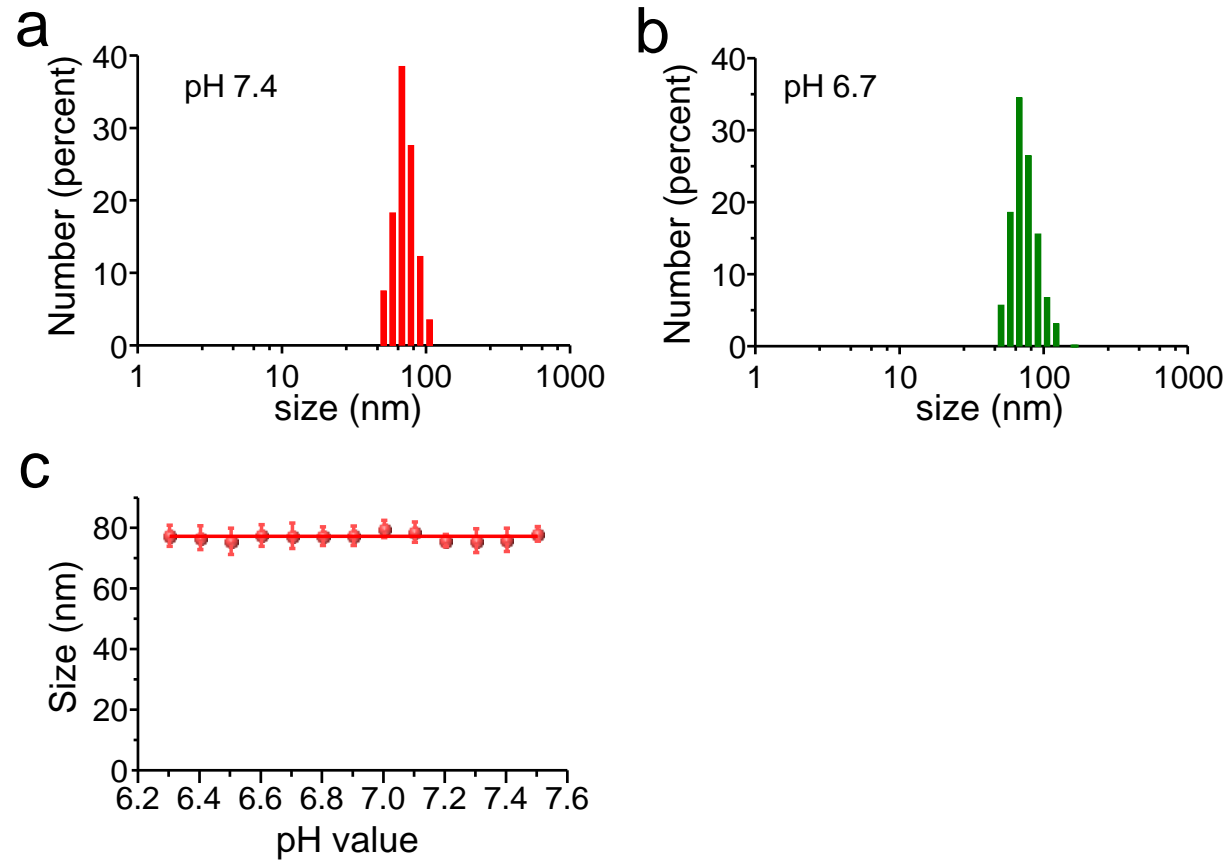

Figure S4. DLS measurements of ICNs/Pt in PB at pH 7.4 (a) and 6.7 (b). (c) Size of ICNs/Pt analyzed by DLS at a series of $\mathrm{pH}$ values from 6.3 to 7.5. 

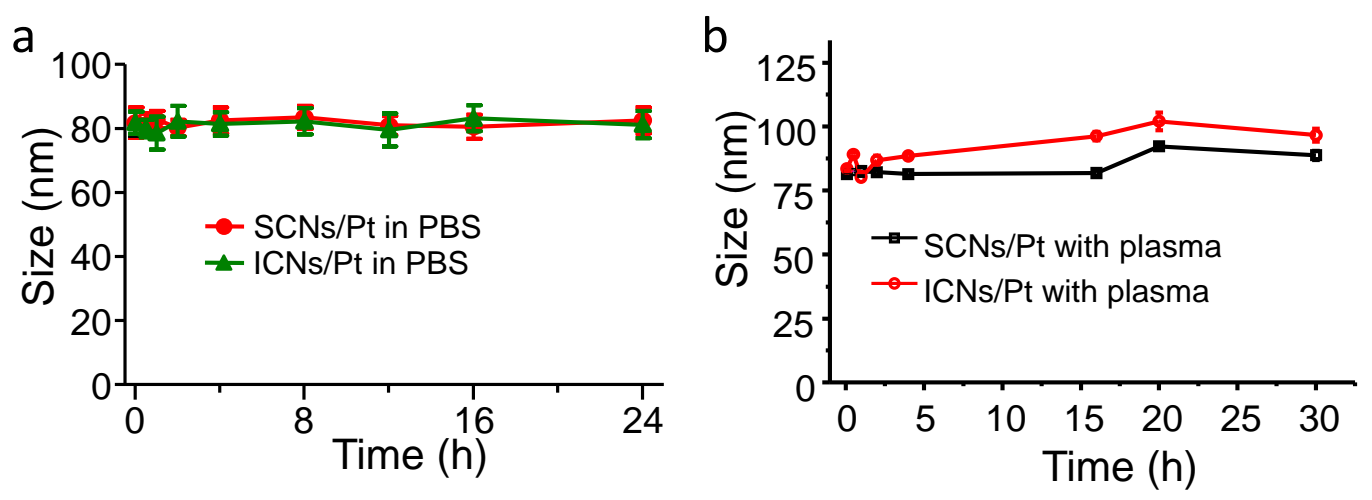

Figure S5. Time-dependent size evolution of SCNs/Pt and ICNs/Pt in PBS at pH 7.4 (a) and in serum (b). Both experiments were carried out at $37^{\circ} \mathrm{C}$, and the nanoparticle concentration was $1 \mathrm{mg} \mathrm{mL}^{-1}$.
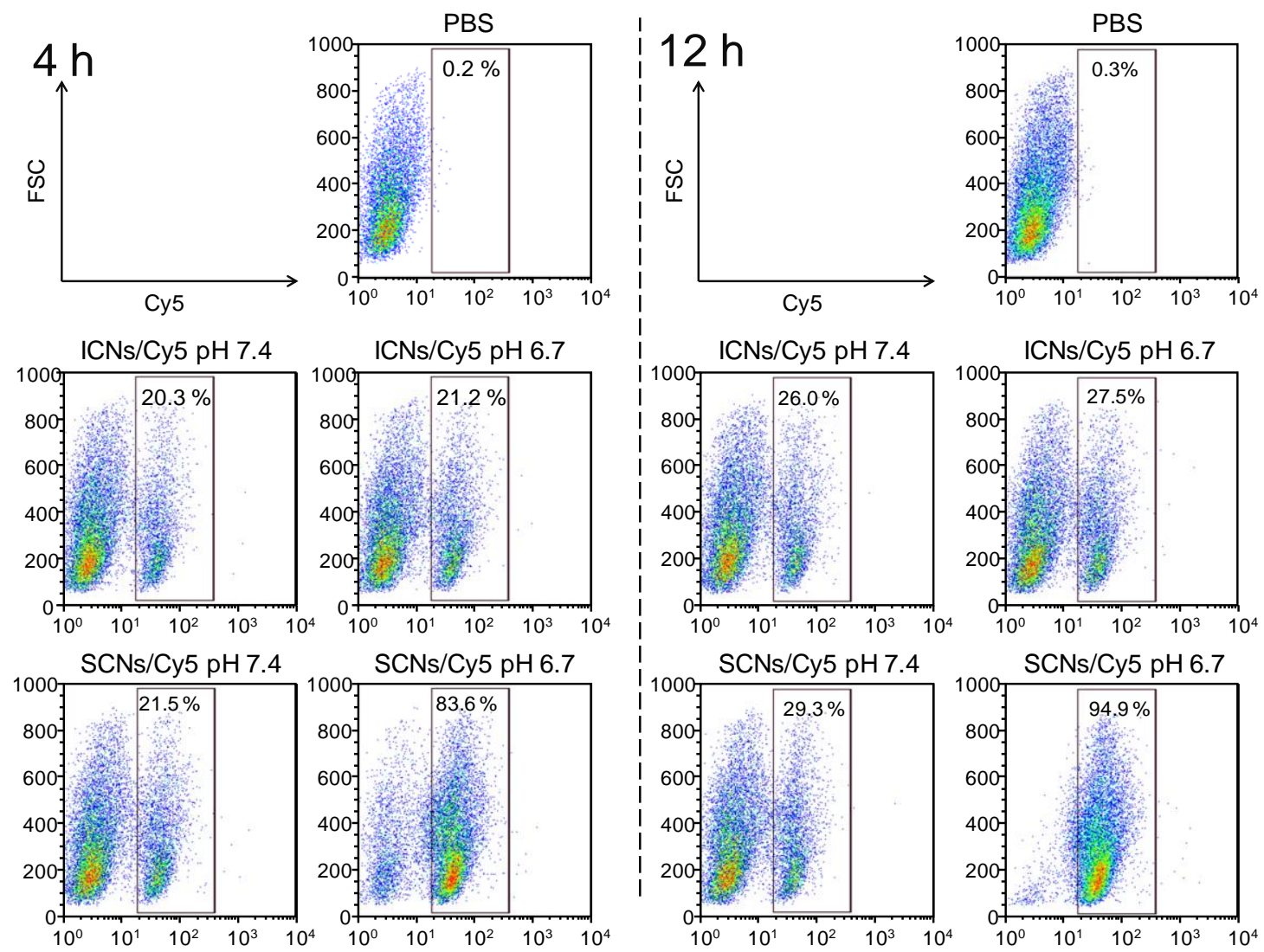

Figure S6. Typical flow cytometry data of MCS cells after incubation with $\mathrm{ICNs} / \mathrm{Cy} 5$ or $\mathrm{SCNs} / \mathrm{Cy} 5$ at $\mathrm{pH} 6.7$ or 7.4 for 4 and $12 \mathrm{~h}$. Cells in the frame were regarded as Cy5-positive cells. 


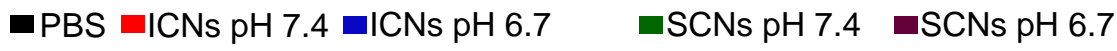
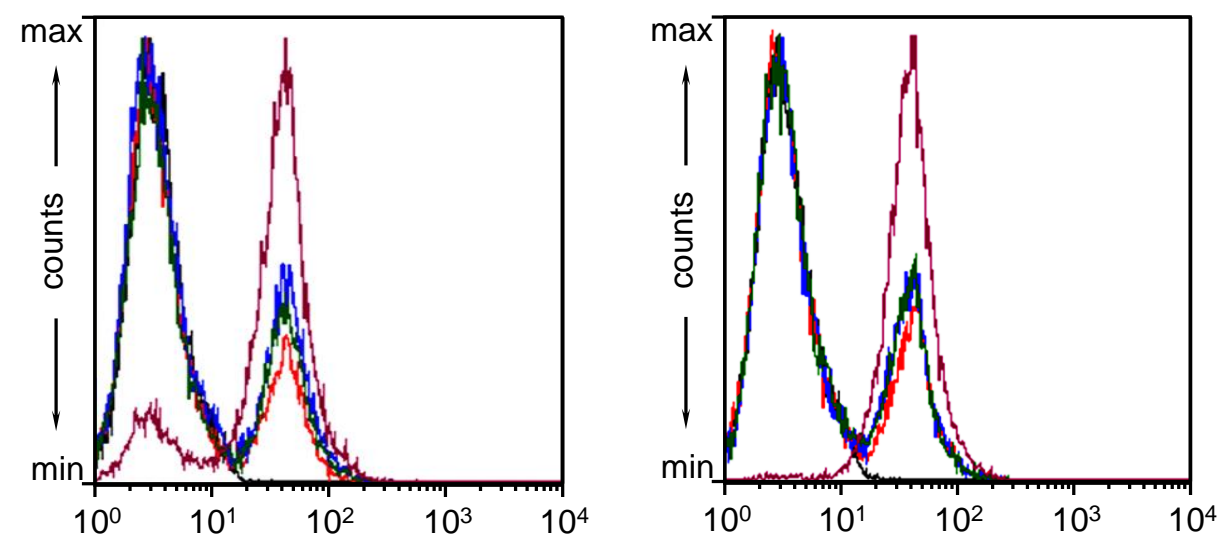

Figure S7. Typical mean fluorescent intensity of flow cytometry data of MCS cells after incubation with ICNs/Cy5 or SCNs/Cy5 at pH 6.7 or 7.4 for $4 \mathrm{~h}$ (left) and $12 \mathrm{~h}$ (right).
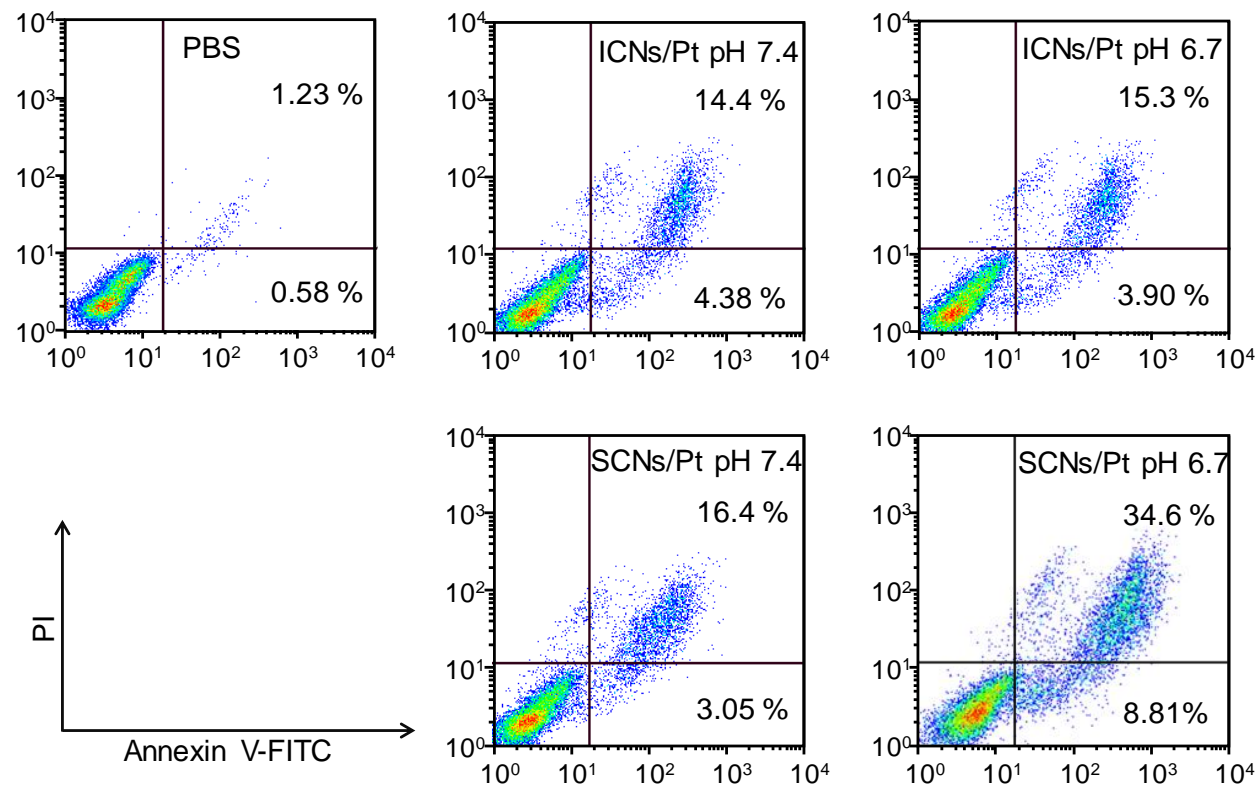

Figure S8. Apoptosis of MCS cells after incubation with different formulations for $24 \mathrm{~h}$ at $\mathrm{pH}$ 6.7 or 7.4. Apoptotic cells were stained with Annexin V-FITC and propidine iodide (PI). 


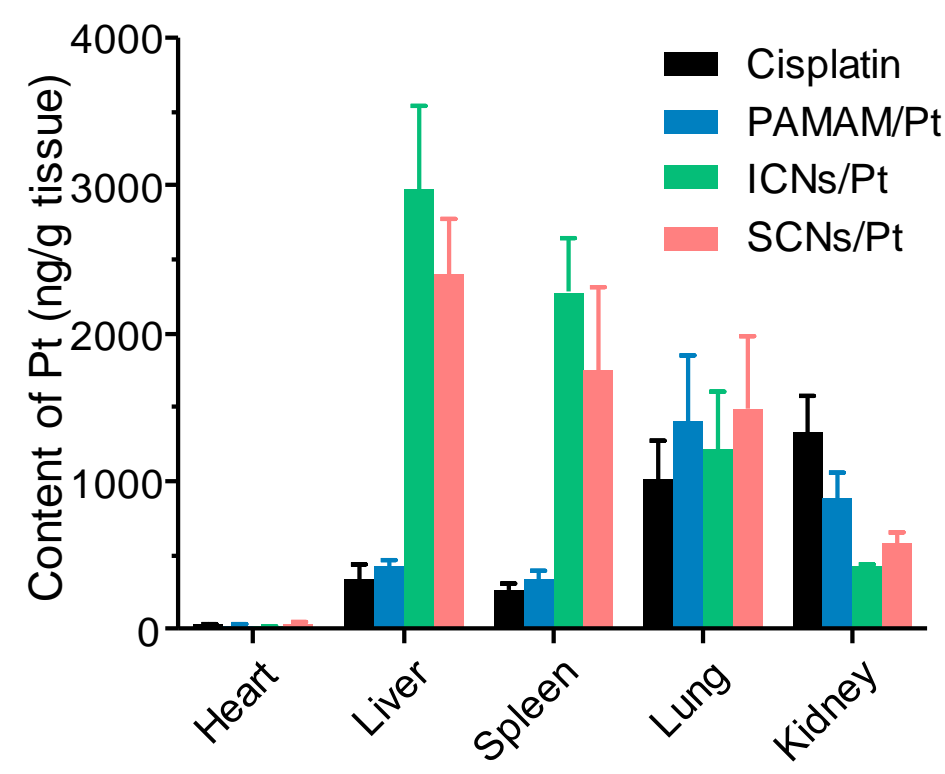

Figure S9. Pt content in major organs at $12 \mathrm{~h}$ after intravenous injection of free cisplatin, PAMAM/Pt, SCNs/Pt, and ICNs/Pt with an equivalent dose of $40 \mu \mathrm{g}$ Pt per mouse. Data are presented as mean $\pm \mathrm{SD}, \mathrm{n}=3$.

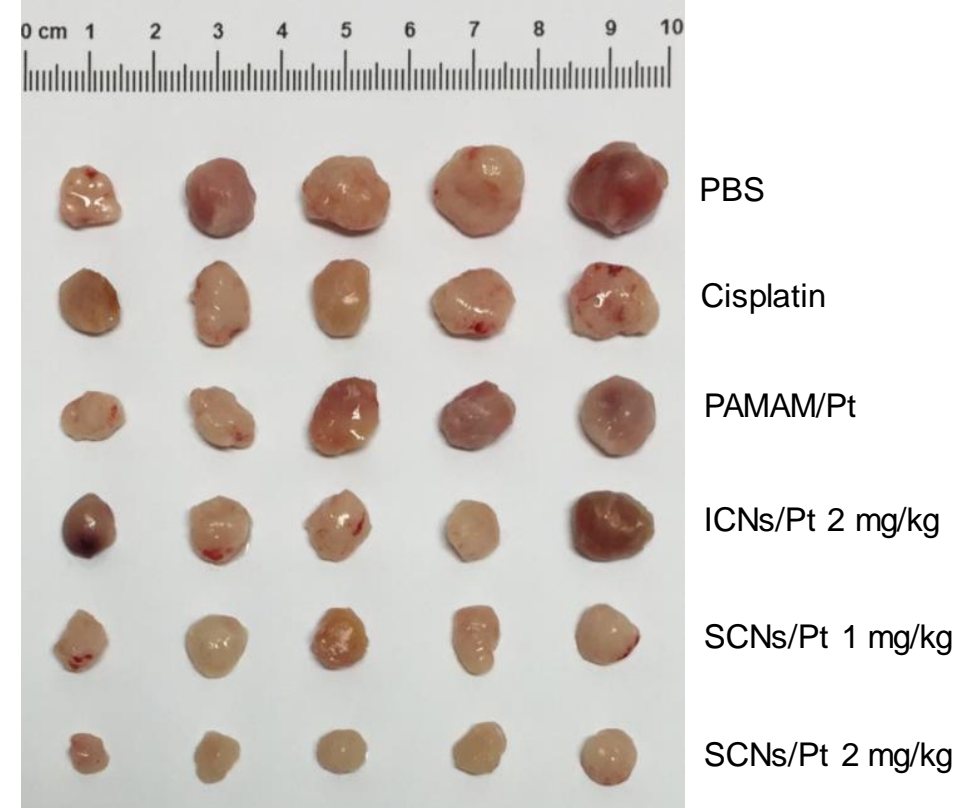

Figure S10. The images of BxPC-3 tumors at the end of treatment. 


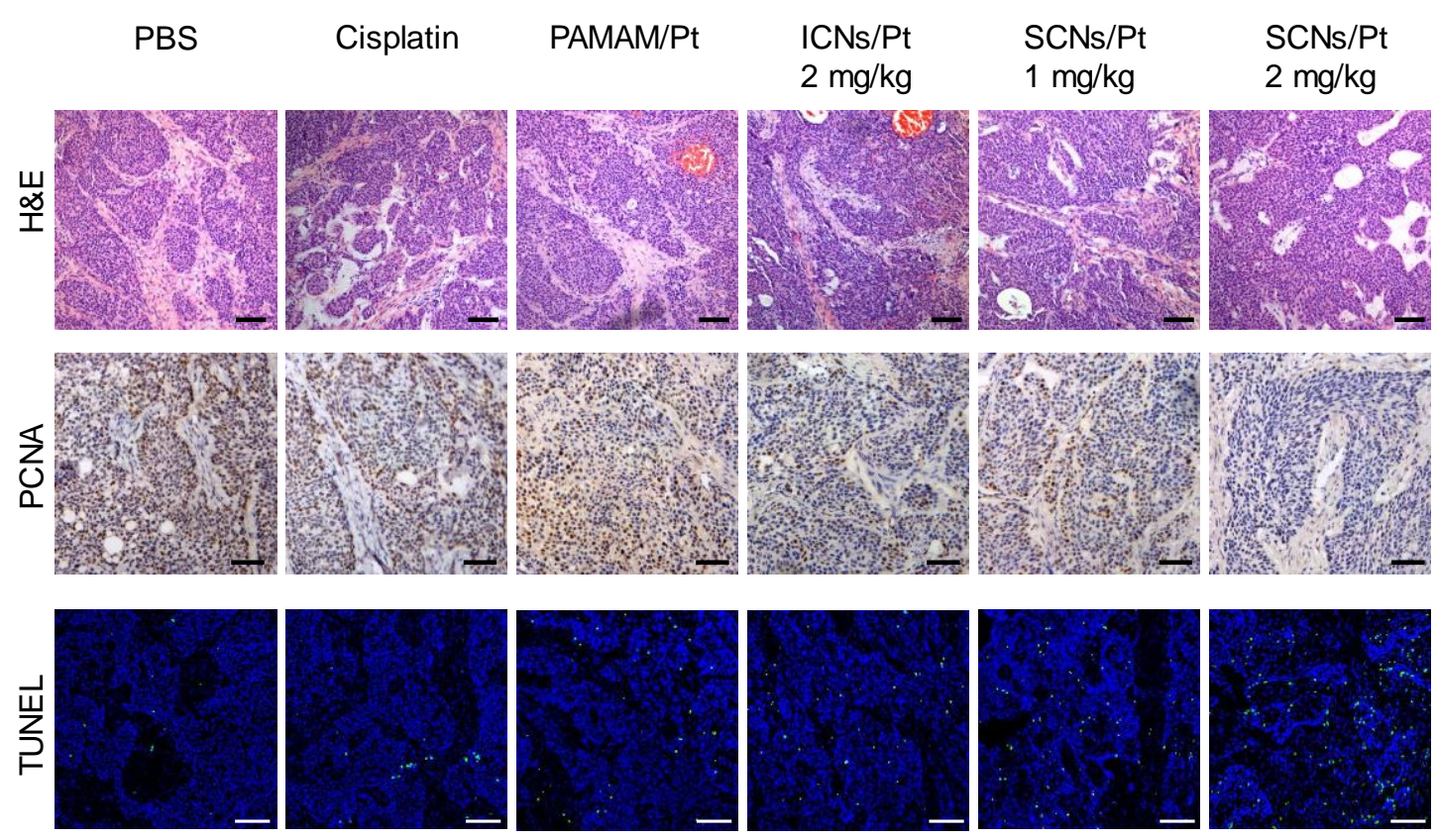

Figure S11. Hematoxylin and eosin (H\&E), proliferating cell nuclear antigen (PCNA), and terminal deoxynucleotidyl transferase-mediated deoxyuridine triphosphate nick end (TUNEL) analyses of BxPC-3 tumor tissues after treatment with various formulations. PCNA-positive proliferating cells are stained brown and TUNEL-positive apoptotic cells are stained green. Scale bar $=100 \mu \mathrm{m}$. 


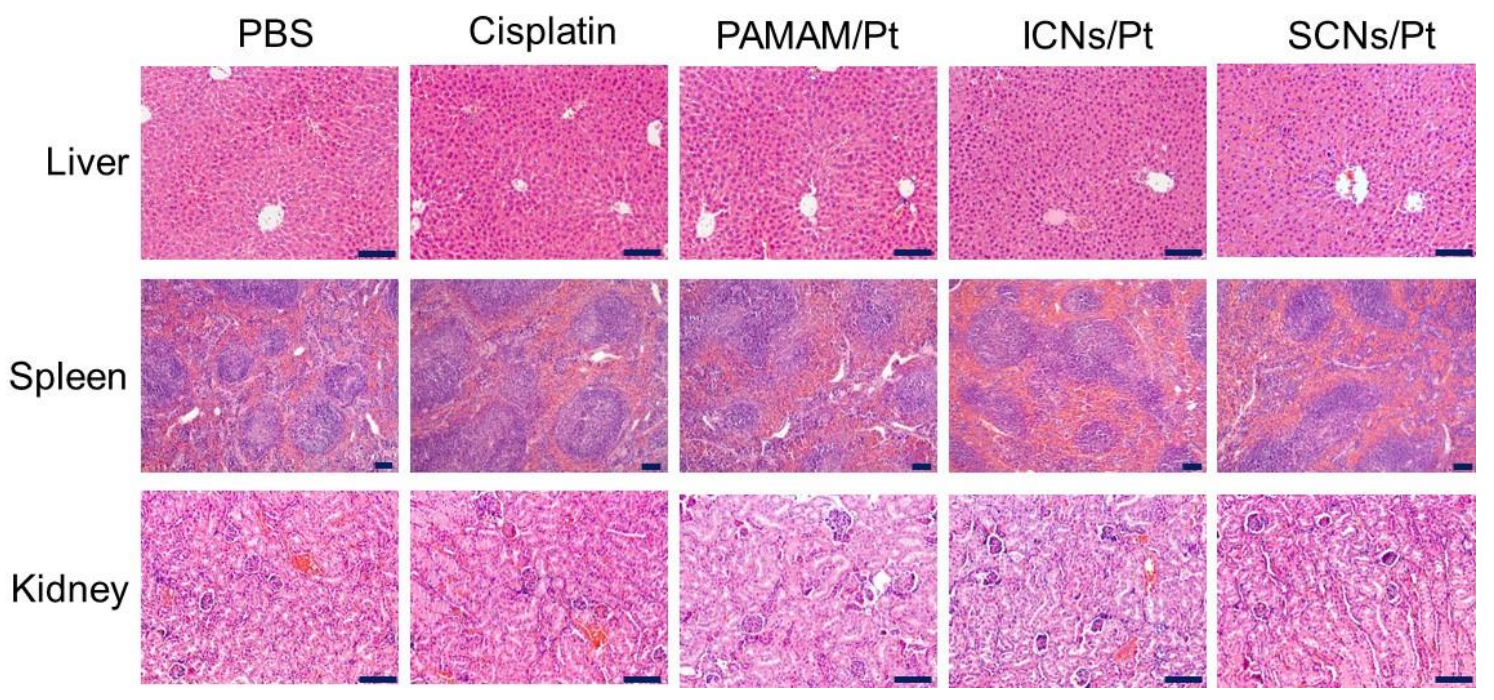

Figure S12. H\&E staining results showing in vivo toxicity of various treatments to major organs such as liver, spleen and kidney after the 21-day tumor inhibition experiment. Scale bar $=100 \mu \mathrm{m}$. 
Table S1. Summary of size, zeta potential, and platinum drug content of SCNs/Pt, ICNs/Pt and PAMAM/Pt.

\begin{tabular}{lccc}
\hline & SCNs/Pt & ICNs/Pt & PAMAM/Pt \\
\hline Size (nm) & $81.3 \pm 3.5$ & $80.1 \pm 2.7$ & $6.2 \pm 0.8$ \\
Zeta potential (mV) & $14.8 \pm 2.1$ & $13.7 \pm 1.9$ & $23.1 \pm 0.6$ \\
Pt content (wt \%) & 2.4 & 2.4 & 9.1 \\
\hline
\end{tabular}

Table S2. Pharmacokinetic parameters of PAMAM/Cy5, SCNs/Cy5, and ICNs/Cy5 after intravenous administration (mean $\pm \mathrm{SD}, \mathrm{n}=3$ ).

\begin{tabular}{|c|c|c|c|c|}
\hline Samples & $\begin{array}{l}T_{1 / 2} \\
\text { (h) }\end{array}$ & $\begin{array}{c}\operatorname{AUC}(0-24 h)^{a} \\
(\mu \mathrm{g} / \mathrm{L} * \mathrm{~h})\end{array}$ & $\begin{array}{l}C_{\max }^{b} \\
(\mu g / L)\end{array}$ & $\begin{array}{c}\operatorname{MRT}(0-24 \text { h) } \\
\text { (h) }\end{array}$ \\
\hline PAMAM/Сy5 & $0.72 \pm 0.07$ & $6.03 \pm 0.56$ & $12.48 \pm 1.47$ & $0.43 \pm 0.04$ \\
\hline $\mathrm{SCNs} / \mathrm{Cy} 5$ & $7.71 \pm 2.24$ & $280.61 \pm 36.82$ & $59.92 \pm 5.48$ & $7.13 \pm 1.08$ \\
\hline ICNs/Cy5 & $7.86 \pm 3.17$ & $267.10 \pm 41.51$ & $58.55 \pm 4.81$ & $7.07 \pm 0.97$ \\
\hline
\end{tabular}

${ }^{\mathrm{a}}$ Area under the curve (AUC).

${ }^{\mathrm{b}}$ The maximum drug concentration $\left(\mathrm{C}_{\max }\right)$.

${ }^{\mathrm{c}}$ Mean residence time (MRT).

The pharmacokinetic parameters were analyzed according to a non-compartment model by DAS 3.0. 